\title{
Research of Status Monitoring System for Special Equipment Storage and Transportation Based on ZigBee
}

\author{
Le Wang $^{1, a_{*}}$, Guanglong Wang ${ }^{1, b_{*}}$,Zhongtao Qiao ${ }^{1, c}$ and Fengqi Gao ${ }^{1, d}$ \\ ${ }^{1}$ Laboratory of Nanotechnology and Microsystems, College of Mechanical Engineering, \\ Shijiazhuang, China, 050003. \\ aliumangtuwl@163.com, bglwang2005@163.com, cqzt7213@sohu.com, dgfq2013@163.com
}

Keywords: ZigBee technology, status monitoring system, ARM, wireless network

\begin{abstract}
A novel of embedded storage and transportation status monitoring system and sampling terminal node based on ZigBee and embedded technology is designed to acquisition the status parameters of the special equipment remotely in this paper. Wireless transmission network is established by ZigBee technology to transfer monitoring data and the data can be processed rapidly and displayed in real-time by ARM microprocessor. The system has the characteristics of low power consumption, high stability, high flexibility and expansibility.
\end{abstract}

\section{Introduction}

Status monitoring technology is the use of modern sensor technology, computer technology to monitor the operational status of the internal and external environment and some of the key features of parameters of special equipment. The monitoring system can monitor parameter status values of the special equipment and storage the monitoring data to assess the health and provide alarms and troubleshooting information when necessary ${ }^{[1]}$. In the data transmission mode, the current storage and transportation status monitoring system is basically connected by cable. However, when the line erection difficulties, corrosive, explosive, and the acquisition target are moving, wired applications will be limited. In this case, the use of wireless transmitted is an alternative pattern. The ZigBee wireless network which has grate network capacity, low power consumption and long battery life is more suitable for wireless status monitoring system ${ }^{[2]}$. Therefore, this paper design the embedded status monitoring system for special equipment storage and transportation by ZigBee technology, compared to traditional embedded storage and transportation status monitoring system, the biggest difference is the intelligence structure, which supports wireless sensor networks and the collecting data toward the direction of high real-time, multi-parameter, high-precision, while data storage become large capacity, more miniaturization and portable, and the development of multi-communication mode and long-distance for data transmission.

\section{Design of the Status Monitoring System}

Design of Hardware Framework of System. The status monitoring system can be roughly divided into four part, they are the terminal monitoring node, routing nodes, ZigBee coordinator and handheld monitoring center. Terminal monitoring node ,which is composed of ZigBee wireless transmission module and monitoring sensor module, is responsible for sampling the data of temperature, humidity, acceleration, pressure, etc. After data collection, format conversion, the data will be transferred between ZigBee collection node and the ZigBee coordinator node. Routers are used to search for the optimal routing path, forwarding data, and as a reference node in positioning system. ZigBee coordinator is responsible for establishing, maintaining, and managing a wireless network, and collecting all the data from the terminal equipment, then, two-way communication with the handheld monitoring center through the serial port ${ }^{[3][4]}$. The user can observe the monitoring of data through handheld monitoring device in real-time. Hardware circuit which is composed of the wireless sensor node, the coordinator node and control unit, is shown in Fig. 1. 
Wireless sensor node

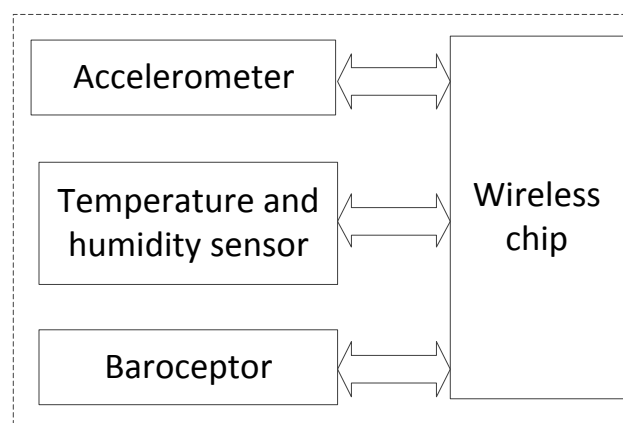

Coordinator node

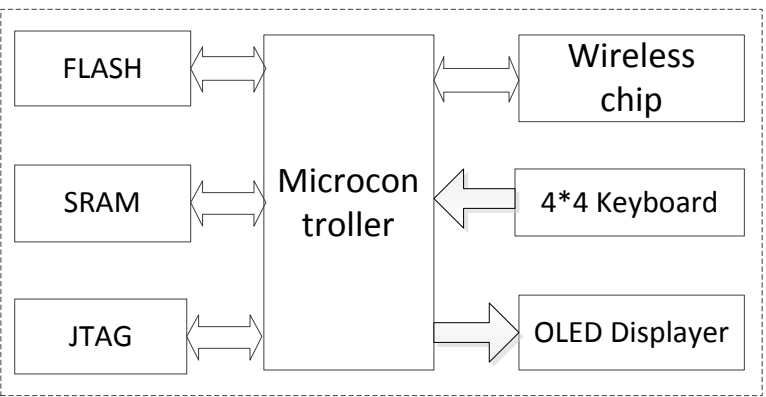

Fig.1.Hardware Framework of System

Design of the Master Circuit. The system core processor is an embedded chip named STM32F4X which has enhanced performance, low cost, low-power. Real-time data acquisition is needed that we have designed the system with $64 \mathrm{~K} * 12$ bit capacity on chip SRAM. This process is connecting the TX and RX on wireless chip with TXD and RXD on USART in the ARM chip, it will start to collect data which is transferred from terminal monitoring node, then the data transmitted through the DMA and stored in the SRAM chip until the data buffer is full, it sends a interrupt signal to CPU, and CPU read all the channels data from FIFO, the result display in the OLED directly ${ }^{[5]}$.

Design of the ZigBee Wireless Communication Circuit. Temperature and humidity, acceleration, and pressure and other data are transferred via ZigBee wireless network. The wireless core chip in the ZigBee wireless transmission network, it can be a very low total cost of materials to establish a strong network node, the interior of the chip has enhanced microcontroller core, compatible 2.4 GHz, IEEE802.15.4 and ZigBee protocol chip technology. Sensor unit connect with the wireless chip directly to become wireless sensor node. After the information was collected, data will be transferred from routing nodes to the coordinator node. The coordinator node and routing nodes on the composition of hardware circuit is consistent.

\section{Data Analysis and Process Method}

The digital temperature and humidity sensor SHT11 be used in the system to realize the detection of temperature and humidity. The sensor has built-in temperature sensor, humidity sensor, signal transformation, A/D conversion, and heater, and other functions module. In order to gain accurate data, we use the following formula modified output values.

$$
\begin{aligned}
& R H_{\text {linear }}=c_{1}+C_{2} \cdot S O_{R H}+C_{3} \cdot S O_{R H}{ }^{2} \\
& R H_{\text {true }}=\left(T_{{ }^{\circ}}{ }_{C}-25\right) \cdot\left(t_{1}+t_{2} \cdot S O_{R H}\right)+R H_{\text {linear }} \\
& T=d_{1}+d_{2} \cdot S O_{T}
\end{aligned}
$$

while the $R H_{\text {linear }}$ for temperature compensation before relative humidity value, $R H_{\text {ture }}$ for relative humidity value after temperature compensation , $\mathrm{SO}_{T}$ for the temperature of the temperature and humidity sensor readout numerical values.

In this paper, to acquire the acceleration parameter, we have used a 16-bit acceleration sensor which is launched by the STMicroelectronics company. This acceleration sensor named LIS302DL with high performance and high stability could collect the data from axis $\mathrm{x}, \mathrm{y}$, and $\mathrm{z}$. In data acquisition process of our system, the error of sampling stage is mainly caused by quantified, we use the following formula calculate the SNR.

$$
\frac{S}{N}=\frac{V \max ^{2}}{e^{2}}=\frac{12 V \max ^{2}}{q^{2}}=12 \times 2^{2 n}
$$

BMP085 is a new generation of high precision pressure sensor. Test pressure and elevation, the first is the controller from pressure and elevation transformation command, then after a period of time the controller from the UP register read the raw data. Finally, need to get the data, the data stored in 
the pressure sensor in the block of EEPROM, can be read through the $\mathrm{I}^{2} \mathrm{C}$. We use the following formula to get the real value of pressure and altitude.

$$
\begin{aligned}
& H_{\text {altitude }}=44330 *\left[1-\left(p / p_{0}\right)^{1 / 5.255}\right] \\
& p=p_{0} /\left(1-\frac{H_{\text {altitude }}}{44330}\right)^{5.255}
\end{aligned}
$$

while $p_{0}=101325 \mathrm{kPa}$, it is atmosphere.

\section{Design of the Software System}

According to the design features, technical scheme and function of each module hardware circuit, the system software consists of the monitoring center module, the coordinator module, sensor information acquisition module. The monitoring center main function is to query information, store data and display the data in real-time and the flow chart is shown in fig.2 (a). The role of the coordinator is to form a network in the system. After the power, coordinator will be initialized and establish the network, for the whole system to establish a legal sensor acquisition node, and then, send and receive the sample data. After establish network successfully, the coordinator play the role of transferring data, the specific flow chart as shown in figure 2(b)[6]. Sensor node is responsible for communicate with the coordinator node and sent the sample data to the coordinator and the flow chart is shown in fig.2(c). The first is the micro system initialization, then the initialization is completed, the coordinator will be started to build the ZigBee network and waiting for the nodes to join the network. When a sampling task is created, sensor nodes will enter working state quickly. First, sensor nodes response to the data sampling command directly, and then start the sensors, collect data and send data. The data will be transferred to monitoring center and displayed on the OLED screen.

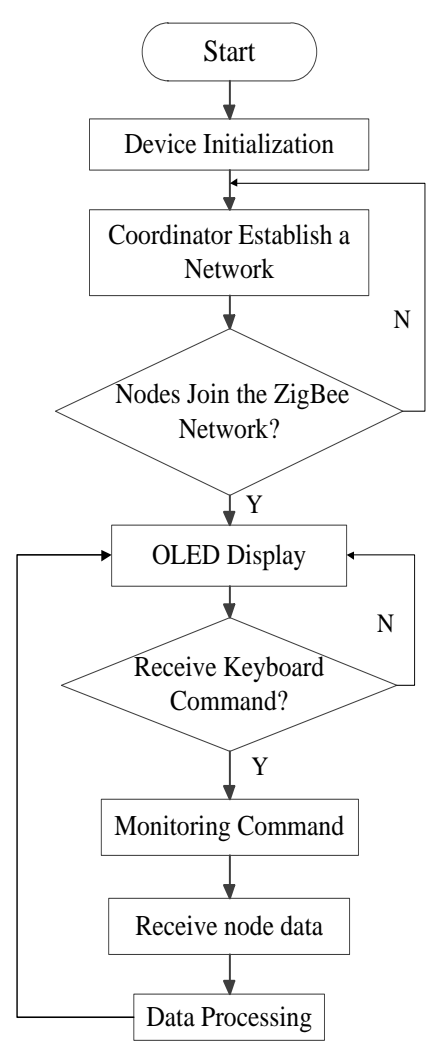

(a)

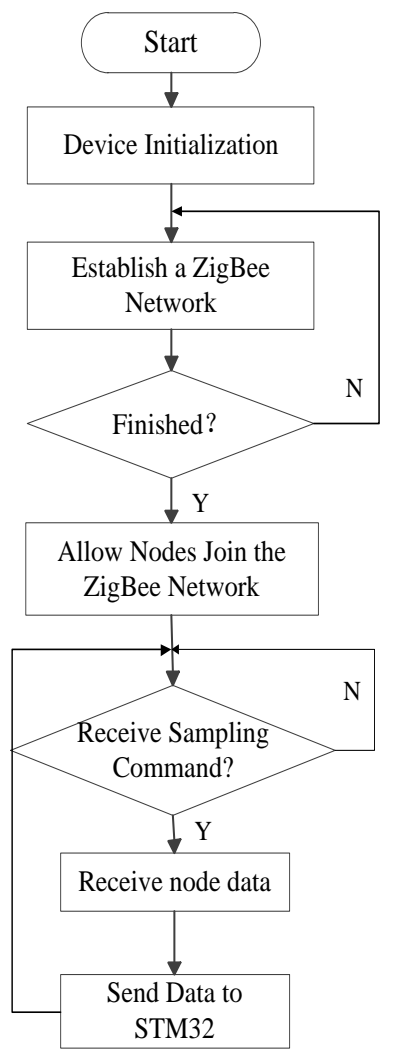

(b)

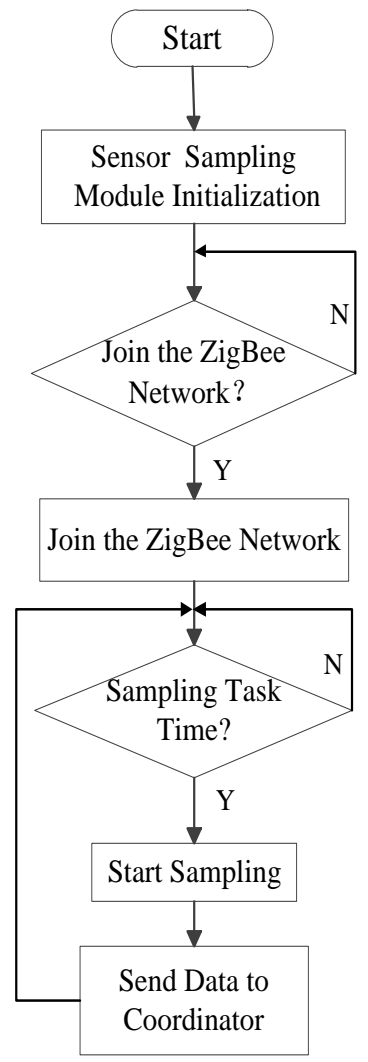

(c)

Fig. 2. (a) Flow chart of the monitoring center (b) Flow chart of the coordinator

(c) Flow chart of the wireless sensor 


\section{Experiment Results}

According to the software and hardware design scheme of the system, we established a sensor data collection network, the network is composed of a ZigBee coordinator node and three terminal nodes whose core is wireless chip. After system initialization, the coordinator will establish a ZigBee network and the terminal nodes join to the network. After a while, the menu is displayed in the OLED. Then, Press F1 key, x-axis, y-axis, z-axis value will be displayed on the OLED screen. When the board is placed horizontally, the $\mathrm{x}$-axis, $\mathrm{y}$-axis, $\mathrm{z}$-axis respectively $+1.64 \mathrm{~g},+0.00 \mathrm{~g},+1.04 \mathrm{~g}$, is shown in fig. 3(a). Press F2 key, microcontroller perform temperature and humidity measurement program. The actual temperature and humidity is shown in fig.3 (b). Press F3 key, microcontroller perform pressure and altitude measurement procedures. The actual pressure and altitude is shown in fig.3(c).The result shows that our system can realize Wireless data transmission and display data in real-time rapidly.

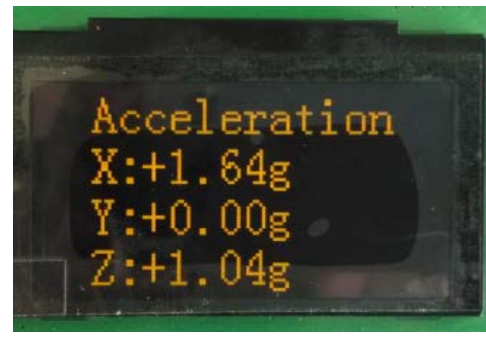

(a)

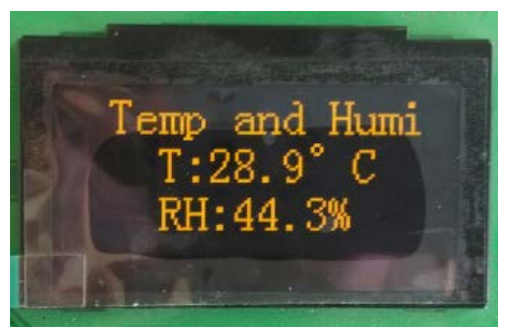

(b)

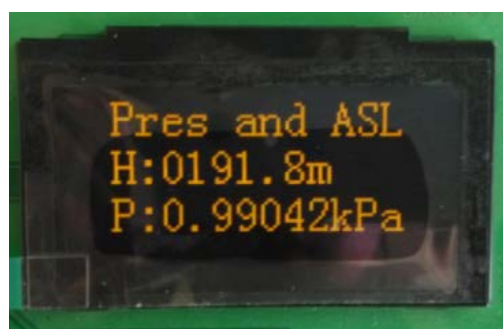

(c)

Fig. 3. (a) The interface of acceleration (b) The interface of temperature and humidity

(c) The interface of pressure and altitude

\section{Summary}

This paper presents a wireless real-time status monitoring system for special equipment storage and transportation based on ZigBee, and introduced the hardware and software structure of the system. It proved that the system can accurately monitor the temperature and humidity, acceleration, pressure and other data. The system can be operated steadily, and has the advantages of flexible networking, good scalability and can be real-time displayed. The experiments show that the system for signal acquisition and wireless transmission is with high reliability and stability, and has development and application value.

\section{References}

[1]. Sushabhan Choudhury, Piyush Kuchhal, Anita. J.ZigBee and Bluetooth Network based Sensory Data Acquisition System. Procedia Computer Science. Vol. 48 (2015), p. 367-372.

[2]. Kunho Hong, SuKyoung Lee. J. Performance improvement in ZigBee-based home networks with coexisting. Pervasive and Mobile Computing. Vol. 19 (2015), p. 156-166.

[3]. Woong Hee Kim, Sunyoung Lee. J. Real-time Energy Monitoring and Controlling System based on ZigBee Sensor Networks. Vol. 5 (2011), p. 794-797.

[4]. Mohammad Ali Moridia, mohammad. J. An investigation of underground monitoring and communication system based on radio waves attenuation using ZigBee. Tunneling and Underground Space Technology. Vol. 43 (2014), p. 362-369.

[5]. Farihah Shariffa. J. Zigbee-based data acquisition system for online monitoring of grid-connected photovoltaic system. Expert Systems with Applications. Vol. 42 (2015) No. 3, p. 1730-1742. 